\title{
Variabilidade genética de duas procedências de mogno africano (Khaya ivorensis $A$. Chev) no cerrado
}

\author{
Genetic variability of two provenances of African mahogany \\ (Khaya ivorensis A. Chev) in the cerrado \\ Lucas Guilherme Moura Oliveira', Poliana Coqueiro Dias' ${ }^{2}$, \\ Edvânio José Gonçalves ${ }^{3}$, Jessika Rodrigues Soares ${ }^{3}$ e Leandro Silva Oliveira ${ }^{3}$
}

\begin{abstract}
RESUMO
A demanda por produtos madeireiros, especialmente de madeiras nobres, tem exercido pressão sobre as formações florestais naturais. Neste contexto, o mogno africano (Khaya ivorensis) apresenta-se como uma alternativa promissora para atender o mercado de madeiras nobres, uma vez que, possui propriedades tecnológicas favoráveis para a produção madeireira. Entretanto, há carência de estudos de melhoramento florestal a respeito da espécie, limitando a seleção de genótipos superiores para a produção de madeira serrada. Portanto, o trabalho teve como objetivo, avaliar a variabilidade genética de caracteres quantitativos, estimando parâmetros genéticos e realizar a predição de valores genéticos dos indivíduos de duas procedências (Mateus Leme e Matosinhos) de K. ivorensis no Cerrado, aos oito anos. O teste foi estabelecido no delineamento experimental em blocos casualizados, com 2 blocos e 49 plantas por parcela. Foram avaliados os caracteres altura total (HT) e diâmetro a altura do peito (DAP). Os componentes de variância e valores genéticos preditos para as variáveis HT e DAP foram estimados utilizando estimadores REML/BLUP. A variância genotípica para as variáveis estudadas apresentou baixa magnitude $(H T=0,34$; $D A P=0,48)$ comparado à variância fenotípica. As variáveis HT e DAP apresentaram, respectivamente, valores de herdabilidade entre famílias de 0,75 e 0,74, associados a valores de acurácia de 0,86 e 0,87, respectivamente. Os ganhos genéticos foram consideráveis para a seleção de árvores matrizes de K. ivorensis para HT e DAP indicando-as como variáveis promissoras na seleção de genótipos.
\end{abstract}

Palavras-chave: Madeira nobre; Melhoramento Florestal; Análise genética; Predição de ganhos.

\begin{abstract}
The demand for timber products, especially of noble woods, has put pressure on native forests. Thus, African mahogany (Khaya ivorensis) represents a promising alternative to meet the market of noble woods, because of its favorable technological properties for timber production. However, there is a lack of studies of tree breeding in this species, limiting the selection of superior genotypes for the production of sawn wood. This study aimed to evaluate the genetic variability, estimating genetic parameters and predicting individual breeding values in two provenances (Mateus Leme and Matosinhos) of K. ivorensis in the Brazilian cerrado (savanna) biome, aged eight years. The trial was established in randomized complete block design, two blocks and 49 plants per plot. The traits, total height (HT) and diameter at breast height (DAP). The components of variance and genetic values predicted for HT and DAP traits were estimated through the REML/BLUP procedure. The genotypic variance for the studied variables presented a low magnitude $(H T=0.34, D A P=0.48)$ compared to the phenotypic variance. The heritability coefficients among families were of the order of 0.75 and 0.74 for the traits HT and DAP, respectively. The accuracy estimates for HT and DAP traits were 0.86 and 0.87 , respectively. The genetic gains were considerable for the selection of superior trees of $K$. ivorensis for the traits $\mathrm{HT}$ and $\mathrm{DBH}$, indicating them as promising traits in the genotypes selection.
\end{abstract}

Keywords: Noble wood; Forest tree breeding; Forestry; Genetic analysis; Predicting gain.

1. Universidade Federal dos Vales do Jequitinhonha e Mucuri - UFVJM. Teófilo Otoni, MG, Brasil.

2. Universidade Federal Rural do Semi-Árido - UFERSA. Mossoró, RN, Brasil.

3. Instituto de Ciências Agrárias, Universidade Federal de Minas Gerais - UFMG. Montes Claros, MG, Brasil.

* Autor correspondente: lucasguilhermemo@hotmail.com 


\section{INTRODUÇÃO}

O Brasil possui um total de 7,84 milhões de hectares de área plantada com essências florestais, para fins madeireiros e não madeireiros (IBÁ, 2017). Apesar dessa área plantada, o desmatamento ilegal e a exploração irregular de espécies de alto valor econômico continua sendo um problema. Ademais, a expansão urbana e da atividade agropecuária tem provocado degradação dos ecossistemas, elevando o risco de extinção de várias espécies (GOMES, 2010; NOVICK et al., 2003). Diante deste cenário há perda de diversidade genética das espécies florestais, tendo implicações como diminuição da sustentabilidade das espécies, aumento das chances de ocorrência de endogamia e redução da habilidade de sobrevivência perante as mudanças climáticas (SOARES, 2014).

As florestas plantadas representam uma alternativa de suprimento da demanda por madeira e na preservação das florestas naturais (LONDRES, 2011; NATIVIDADE, 2016; SILVA, 2013). Com isto, tem se buscado alternativas para a produção de madeiras nobres, diminuindo a pressão exercida sob as florestas naturais (IBF, 2017a).

O mogno africano (Khaya ivorensis) é uma espécie que apresenta um considerável crescimento, com alta produtividade, boa adaptabilidade, resistência a broca-das-meliáceas (Hypsipyla grandella) e cuja madeira tem qualidade e alto valor no mercado internacional, o que fomentou a expansão do seu plantio por todo o território brasileiro (FARIAS et al. 2011; FIGUEIREDO; OLIVEIRA; BARBOSA, 2005; GOMES, 2010; NATIVIDADE, 2016; ROSA, 2014; TEIXEIRA, 2011). Atualmente, os plantios de K. ivorensis perfazem cerca de 10 mil hectares no Brasil, tendo a metade plantado na região norte de Minas Gerais, sendo destinados a produção de madeira serrada (BARROS; SILVA; PANDOLFI, 2017; IBF, 2017b).

Entretanto, há carência de informações a respeito do manejo silvicultural da espécie, características tecnológicas da madeira e sobre a diversidade genética das populações que constituem os plantios florestais de K. ivorensis. Portanto, esse fator é limitante para avanços quanto ao melhoramento genético da espécie visando ganhos genéticos para características de crescimento, forma, resistência a pragas e patógenos e qualidade da madeira (SILVA, 2015; SOARES, 2014).

Os estudos a respeito da diversidade genética das populações de K. ivorensis possibilitam obter informações quanto a estrutura genética das mesmas, e assim definir estratégias de conservação e aumento da sua variabilidade genética para exploração em programas de melhoramento florestal. Para tanto, para a seleção de materiais genéticos superiores por meio da utilização de modelos mistos, através da metodologia Reml/Blup (máxima verossimilhança restrita/melhor predição linear não viesada) obtêm-se estimativas da estrutura genética de uma população, com a predição de valores genéticos e ganhos com seleção (RESENDE, 2007).

O presente trabalho teve como objetivo estimar a variabilidade genética de duas procedências de K. ivorensis, concomitantemente a seleção genotípica das melhores matrizes em um plantio com 8 anos de idade, no Cerrado na região norte de Minas Gerais pela estimativa dos parâmetros genéticos de variáveis quantitativas de crescimento em diâmetro, altura e forma do tronco.

\section{MATERIAL E MÉTODOS}

\section{Localização e caracterização da fazenda}

O trabalho foi conduzido na Fazenda Retiro Novo, no município de Corinto, região norte de Minas Gerais ( $18^{\circ} 25^{\prime} 37.62^{\prime \prime} \mathrm{S}$; $44^{\circ} 26^{\prime} 1.49^{\prime \prime} \mathrm{O}$; altitude 673m).

A fazenda possui uma área de 245 ha onde aproximadamente 180 ha são ocupados com plantios de K. ivorensis, com 4, 6, 7 e 8 anos de idade. A região é caracterizada como clima tropical, Aw segundo a classificação climática de Koppen. A temperatura média anual em Corinto é de $22,4^{\circ} \mathrm{C}$. A umidade relativa média do ar é de $62,41 \%$ e a pluviosidade média de $1.157 \mathrm{~mm} / \mathrm{ano}$, tendo uma diferença de precipitação de aproximadamente $283 \mathrm{~mm}$ entre o mês mais seco (agosto) e o mês mais chuvoso (dezembro) (CLIMATE-DATA, 2017).

Anteriormente à realização do plantio, foi realizada a incorporação com grade de 2,0 toneladas/ha de calcário. O espaçamento empregado para o plantio foi de 5,25 x 5,25 m. O plantio das mudas foi realizado em covas com $60 \mathrm{~cm}$ de profundidade, utilizando para o preparo das mesmas motocoveadora 
Oliveira et al. - Variabilidade genética de duas procedências de mogno

africano (Khaya ivorensis A. Chev) no cerrado

manual. A adubação de plantio constituiu na aplicação de 150g de NPK 10-30-16 por cova, além da aplicação do hidrogel na proporção de 1,0 kg para cada mil mudas. Os tratos silviculturais pós-plantio consistiram no controle da matocompetição, por meio de roçada semi mecanizada.

\section{Amostragem de campo e delineamento experimental}

O estudo foi conduzido utilizando duas procedências de K. ivorensis, Mateus Leme e Matosinhos, com 8 anos de idade, plantadas em duas áreas de 16,7 ha e 4,10 ha respectivamente. A coleta de dados foi realizada em dois blocos por procedência plantada, distribuídos conforme a regularidade da superfície do terreno dentro da área plantada.

Foram mesurados os dados dendrométricos, altura total (HT) e a circunferência a 1,30 m (CAP), de todas as árvores das parcelas. As mensurações da HT foram realizadas utilizando um clinômetro digital Haglof e uma fita métrica, afim de regular a distância entre o operador e a árvore a ser medida. O CAP foi mensurado utilizando uma fita métrica medindo as circunferências de forma perpendicular ao eixo da árvore, na altura de 1,30 m e posteriormente estes valores de CAP foram transformados, para obtenção dos valores de diâmetro (DAP) à 1,30 m do solo.

O experimento foi conduzido no delineamento de blocos casualizados (DBC), com 2 tratamentos (duas procedências de K. ivorensis - Mateus Leme e Matosinhos) e 2 blocos, sendo cada bloco composto por duas parcelas, cada uma com 49 árvores (36 x 36 m).

\section{Estimativas de parâmetros genéticos e estatística}

As estimativas dos componentes de variância e predição de valores genéticos foi realizada utilizando o procedimento REML/BLUP (estimativa de componentes de variância - RELM e de predição de valores genéticos - BLUP). Para a avaliação foi testado o a pressuposição de homogeneidade das variáveis HT e DAP e posteriormente a demais análises estatísticas foram realizadas utilizado o software Selegen - REML/BLUP.

As duas procedências estudas foram avaliadas por dois modelos estatísticos. O modelo denominado 24 pelo Selegen que trata as procedências como de efeito aleatório e o modelo 97 que trata as procedências como de efeito fixo. Conforme Resende e Duarte (2007), fatores com 5 ou mais níveis devem preferencialmente ser considerados como de efeitos aleatórios. Assim, no presente estudo faz-se uma comparação considerando as procedências como de efeito aleatório e de efeito fixo.

Os dados para a avaliação das árvores de K. ivorensis, foram avaliados através de dois modelos lineares mistos univariados (considerando as procedências como efeito aleatório e outro considerando-as como de efeito fixo) do software Selegen - REML/BLUP, apresentado por Resende, (2007):

Modelo 24, considerando as procedências como efeito aleatório.

$$
y=X r+Z g+W p+e
$$

Onde,

$\mathrm{y}=$ vetor de dados;

$\mathrm{r}=$ vetor dos efeitos de repetição (assumidos como fixos) somados à média geral;

$\mathrm{g}=$ vetor dos efeitos genotípicos de populações (assumidos como aleatórios);

$\mathrm{p}=$ vetor dos efeitos de parcelas (aleatórios);

e = valor de erros ou resíduos (aleatórios);

$\mathrm{X}, \mathrm{Z}$ e W representam as matrizes de incidência dos referidos efeitos (RESENDE, 2007).

Modelo 97, considerando as procedências como efeito fixo.

$$
y=X t+Z b+W p+e
$$

Onde,

$\mathrm{y}=$ vetor de dados

$\mathrm{t}=$ vetor dos efeitos de tratamentos (assumidos como fixos) somados à média geral

$\mathrm{b}=$ vetor dos efeitos aleatórios de blocos

$\mathrm{p}=$ vetor dos efeitos aleatórios de parcela

$\mathrm{e}=$ vetor de erros ou resíduos (aleatórios).

$\mathrm{X}, \mathrm{Z}$ e W representam as matrizes de incidência dos referidos efeitos (RESENDE, 2007). 


\section{RESULTADOS E DISCUSSÃO}

A variância genotípica das variáveis estudadas apresentou valores de baixa e média magnitude (TABELA 1). A variância genotípica para as variáveis estudadas apresentou baixa magnitude, 0,34 para HT e 0,48 para DAP, comparado à variância fenotípica. As variáveis HT e DAP apresentaram, respectivamente, valores de herdabilidade entre procedências de 0,75 e 0,74. Associados a estas variáveis, estimou-se valores de acurácia de 0,86 e 0,87, para HT e DAP, respectivamente.

A ausência de um efeito genético de alto magnitude sobre as variáveis analisadas pelo modelo de efeito aleatório pode acarretar em baixos ganhos com a seleção, uma vez que, o ganho com seleção é uma função da variabilidade genética total. Também, tem- se a hipótese que os plantios de K. ivorensis são constituídos em sua maioria de sementes de árvores matrizes plantadas em 1976 no Belém do Pará. Diante dessa problemática, caso a mesma se confirme, há a necessidade de introdução de novas procedências de K. ivorensis no país. A baixa variabilidade genética dos plantios também implica em riscos que podem comprometer toda a atividade silvicultural com K. ivorensis. A variabilidade genética em uma população é de suma importância para a sua sustentabilidade, além de ser fator fundamental para a obtenção de ganhos genéticos com a seleção (BERTI, 2010).

Table 1. Estimates of the components of variance for the traits total height (HT) and diameter at breast height $(\mathrm{DBH})$ considering the provenances of $\mathrm{K}$. ivorensis as random effect and fixed effect, at 8-years-old, in the municipality of Corinto - MG.

Tabela 1. Estimativas dos componentes de variância para as variáveis altura total (HT) e diâmetro à altura do peito (DAP) considerando as procedências de K. ivorensis como efeito aleatório e efeito fixo, aos 8 anos de idade, no município de Corinto - MG.

\begin{tabular}{|c|c|c|c|c|}
\hline \multirow[t]{2}{*}{ Estimativas } & \multicolumn{2}{|c|}{$\begin{array}{l}\text { Modelo misto } \\
\text { Efeito aleatório }\end{array}$} & \multicolumn{2}{|c|}{$\begin{array}{l}\text { Modelo misto } \\
\text { Efeito fixo }\end{array}$} \\
\hline & HT & DAP & HT & DAP \\
\hline F (procedência) & - & - & 1,23 & 3,65 \\
\hline$\sigma^{2} b$ & - & - & 0,02 & 0,00 \\
\hline$\sigma^{2} g$ & 0,34 & 0,48 & 1,07 & 0,39 \\
\hline$\sigma^{2}$ parc & 0,34 & 0,48 & 1,05 & 0,38 \\
\hline$\sigma^{2} e$ & 5,08 & 8,86 & 5,11 & 9,13 \\
\hline$\sigma^{2} f$ & 5,75 & 9,82 & 6,18 & 9,52 \\
\hline$b^{2}$ & - & - & 0,00 & 0,00 \\
\hline$c^{2}$ parc & 0,06 & 0,05 & 0,17 & 0,04 \\
\hline $\mathrm{h}^{2} \mathrm{mp}$ & 0,75 & 0,74 & 0,19 & 0,73 \\
\hline Acproc & 0,87 & 0,86 & - & - \\
\hline $\mathrm{CVe} \%$ & 15,67 & 16,82 & 7,46 & 4,27 \\
\hline Média geral & 14,40 & 17,67 & 14,40 & 17,67 \\
\hline
\end{tabular}

$\sigma^{2}$ b: componente de variância entre blocos; $\sigma^{2}$ g: variância genotípica entre populações; $\sigma^{2}$ parc: variância ambiental entre parcelas; $\sigma^{2} e$ : variância residual; $\sigma^{2}$ f: variância fenotípica individual; $b^{2}$ : coeficiente de determinação associado a blocos; $c^{2}$ parc: coeficiente de determinação dos efeitos de parcelas; $h^{2} \mathrm{mp}$ : herdabilidade da média de populações, assumindo sobrevivência completa; Acproc: acurácia da seleção de populações, assumindo sobrevivência completa; CVe\%: coeficiente de variação experimental.

Desta forma, a introdução de novos genótipos torna importante ferramenta para o aumento da variabilidade genética dos povoamentos florestais. Tal ferramenta também possibilita maiores ganhos genéticos para caracteres de interesse, além de benefícios socioeconômicos como o aumento da produtividade advindo da maior adaptabilidade destes genótipos aos diversos fatores edaficoclimáticos presentes no país (SAMPAIO, 2000).

Os altos valores de herdabilidade $\left(\mathrm{h}^{2} \mathrm{mp}\right)$ favorecem a seleção inicial entre progênies, acompanhada pela seleção dentro das melhores progênies selecionadas. Desta forma, foram observadas altas magnitudes de herdabilidade para as variáveis HT e DAP, respectivamente. O progresso esperado ao se realizar a seleção depende diretamente da herdabilidade (RESENDE, 2002), assim, observa-se a possibilidade de ganhos genéticos para as variáveis, HT e DAP.

O valor de acurácia (Acproc) estimado para a variável HT foi igual a 0,87, considerada alta, demonstrando uma alta correlação entre valores genéticos preditos e valores genéticos reais dos genótipos. As maiores magnitudes para a acurácia estão ligadas a maior confiabilidade da avaliação e do valor genético predito de um indivíduo, e também expressa, a qualidade do método de seleção, pois quanto maior for a acurácia, melhor preditor do valor genético verdadeiro é o método de seleção.

Martinez et al., (2012) e Rosado et al., (2009), encontraram resultados de alta magnitude para estimativa da herdabilidade da variável altura $\left(h^{2} \mathrm{mp}=0,71 \mathrm{e} \mathrm{h}^{2} \mathrm{mp}=0,77\right)$ em progênies de $P$. taeda 
e clones de eucalipto respectivamente. Estes autores também encontraram resultados de altas magnitudes para a variável diâmetro $\left(\mathrm{h}^{2} \mathrm{mp}=0,87 \mathrm{e} \mathrm{h}^{2} \mathrm{mp}=0,86\right)$ em progênies de $P$. taeda e clones de eucalipto, respectivamente.

Estas estimativas são semelhantes às encontradas neste trabalho, assim observando um alto controle genético para tais variáveis, justificando a sua utilização no que tange à seleção de genótipos visando ganhos em altura e diâmetro.

Os coeficientes variação encontrados para os dois métodos, se distinguem entre os métodos para as características avaliadas (Tabela 1). O menor coeficiente de variação, é apresentado pelo modelo onde considera as progênies como de efeito fixo, desta forma, demonstrando uma boa precisão experimental, de acordo com a classificação proposta por COUTO et al., (2008).

As variâncias ambientais obtidas pelas duas metodologias, apresentaram-se não muito distantes com exceção da encontrada para a variável HT. Esta diferença pode ser devida à esta variável sofrer forte influência do ambiente. Entretanto, os resultados observados para a variável DAP, são semelhantes entre as duas metodologias, o que indica certa semelhança entre os procedimentos utilizados em relação à forma de obtenção dos parâmetros genéticos, para as variáveis estudadas. As estimativas de média geral, apresentaram-se iguais em ambas as metodologias.

Os resultados dos valores preditos pelo BLUP individual e para seleção fenotípica das variáveis HT e DAP foram ranqueados, desta forma, indicando quais os dez melhores genótipos de K. ivorensis em ambas procedências, utilizando duas diferentes formas de seleção (Tabelas 2 e 3 ).

Table 2. Estimates obtained from the gain with selection of the ten best $K$. ivorensis genotypes evaluated in two methodologies, regarding the total height (HT) trait, at 8-years-old, in the municipality of Corinto - MG.

Tabela 2. Estimativas obtidas do ganho com seleção dos dez melhores genótipos de $K$. ivorensis avaliados em duas metodologias, quanto ao caráter altura total (HT), aos 8 anos de idade, no município de Corinto - MG.

\begin{tabular}{|c|c|c|c|c|c|c|c|}
\hline \multirow{2}{*}{ Ordem } & \multicolumn{7}{|c|}{ Seleção com base no valor genético } \\
\hline & Procedência & Árvore & Parcela & Bloco & $f$ & Ganho & Nova Média \\
\hline 1 & Mateus Leme & 26 & 2 & 2 & 19,40 & 0,83 & 15,21 \\
\hline 2 & Mateus Leme & 35 & 2 & 1 & 18,60 & 0,83 & 15,21 \\
\hline 3 & Mateus Leme & 36 & 2 & 1 & 18,00 & 0,80 & 15,19 \\
\hline 4 & Mateus Leme & 8 & 2 & 1 & 17,90 & 0,79 & 15,18 \\
\hline 5 & Mateus Leme & 38 & 2 & 1 & 17,80 & 0,78 & 15,17 \\
\hline 6 & Mateus Leme & 41 & 2 & 1 & 17,80 & 0,77 & 15,16 \\
\hline 7 & Mateus Leme & 43 & 2 & 1 & 17,80 & 0,77 & 15,15 \\
\hline 8 & Mateus Leme & 46 & 2 & 1 & 17,80 & 0,76 & 15,15 \\
\hline 9 & Mateus Leme & 24 & 2 & 2 & 18,40 & 0,76 & 15,14 \\
\hline 10 & Mateus Leme & 16 & 2 & 2 & 17,50 & 0,75 & 15,14 \\
\hline \multirow{2}{*}{ Ordem } & \multicolumn{7}{|c|}{ Seleção com base no valor fenotípico } \\
\hline & Procedência & Árvore & Parcela & Bloco & f & Ganho & Nova Média \\
\hline 1 & Matosinhos & 36 & 1 & 2 & 19,90 & 4,13 & 18,53 \\
\hline 2 & Mateus Leme & 26 & 2 & 2 & 19,40 & 3,75 & 18,15 \\
\hline 3 & Matosinhos & 45 & 1 & 2 & 19,00 & 3,45 & 17,85 \\
\hline 4 & Mateus Leme & 49 & 1 & 1 & 18,80 & 3,30 & 17,70 \\
\hline 5 & Mateus Leme & 27 & 1 & 1 & 18,70 & 3,23 & 17,63 \\
\hline 6 & Mateus Leme & 35 & 2 & 1 & 18,60 & 3,15 & 17,55 \\
\hline 7 & Mateus Leme & 46 & 1 & 2 & 18,40 & 3,00 & 17,40 \\
\hline 8 & Mateus Leme & 24 & 1 & 2 & 18,40 & 3,00 & 17,40 \\
\hline 9 & Matosinhos & 27 & 1 & 1 & 18,00 & 2,70 & 17,10 \\
\hline 10 & Mateus Leme & 36 & 2 & 1 & 18,00 & 2,70 & 17,10 \\
\hline
\end{tabular}

f: valor fenotípico individual.

As metodologias de seleção, com base no valor genético e com base no valor fenotípico, proporcionaram diferentes ordenamentos dos genótipos. Ao comparar as duas metodologias, observa-se que houveram significativas alterações no ordenamento da metodologia REML/BLUP (Tabela 2). Porém, isso não incluiu os genótipos da procedência Matosinhos para a seleção com base no valor genético. Os melhores genótipos dessa procedência foram assim classificados somente no método com base no valor fenotípico. Ressalta-se, a alteração de posição da árvore de número 26 da parcela 2 do bloco 2, a qual ocupava a primeira posição no método com base no valor genético que foi alterada para a segunda posição no método com base no valor fenotípico (Tabela 2). O mesmo resultado foi observado para a variável DAP, onde a árvore que ocupava a primeira posição, não mais a ocupa pelo método de seleção fenotípica (Tabela 3). 
Table 3. Estimates obtained from the selection of the ten best $K$. ivorensis genotypes evaluated in two methodologies, regarding the diameter at breast height $(\mathrm{DBH})$ trait, at 8-years-old, in the municipality of Corinto - MG.

Tabela 3. Estimativas obtidas do ganho com seleção dos dez melhores genótipos de $K$. ivorensis avaliados em duas metodologias, quanto ao caráter diâmetro a altura do peito (DAP), aos 8 anos de idade, no município de Corinto - MG.

\begin{tabular}{|c|c|c|c|c|c|c|c|}
\hline \multirow{2}{*}{ Ordem } & \multicolumn{7}{|c|}{ Seleção com base no valor genético } \\
\hline & Procedência & Árvore & Parcela & Bloco & $f$ & Ganho & Nova Média \\
\hline 1 & Mateus Leme & 28 & 1 & 1 & 26,42 & 1,14 & 18,81 \\
\hline 2 & Mateus Leme & 35 & 1 & 2 & 25,18 & 1,11 & 18,78 \\
\hline 3 & Mateus Leme & 36 & 1 & 1 & 25,15 & 1,07 & 18,74 \\
\hline 4 & Mateus Leme & 8 & 1 & 2 & 24,35 & 1,05 & 18,72 \\
\hline 5 & Mateus Leme & 38 & 1 & 1 & 24,70 & 1,03 & 18,70 \\
\hline 6 & Mateus Leme & 41 & 2 & 1 & 22,54 & 1,01 & 18,68 \\
\hline 7 & Mateus Leme & 43 & 1 & 2 & 23,49 & 1,00 & 18,67 \\
\hline 8 & Mateus Leme & 46 & 1 & 1 & 24 & 0,98 & 18,65 \\
\hline 9 & Mateus Leme & 24 & 1 & 1 & 24 & 0,97 & 18,64 \\
\hline 10 & Mateus Leme & 16 & 1 & 2 & 23,08 & 0,96 & 18,63 \\
\hline \multirow{2}{*}{ Ordem } & \multicolumn{7}{|c|}{ Seleção com base no valor fenotípico } \\
\hline & Procedência & Árvore & Parcela & Bloco & $f$ & Ganho & Nova Média \\
\hline 1 & Mateus Leme & 36 & 1 & 1 & 26,42 & 6,47 & 24,14 \\
\hline 2 & Mateus Leme & 26 & 1 & 2 & 25,18 & 5,56 & 23,23 \\
\hline 3 & Mateus Leme & 45 & 1 & 1 & 25,15 & 5,53 & 23,20 \\
\hline 4 & Mateus Leme & 49 & 1 & 1 & 24,70 & 5,20 & 22,87 \\
\hline 5 & Mateus Leme & 27 & 1 & 2 & 24,35 & 4,94 & 22,61 \\
\hline 6 & Mateus Leme & 35 & 1 & 1 & 24,00 & 4,68 & 22,35 \\
\hline 7 & Mateus Leme & 46 & 1 & 1 & 24,00 & 4,68 & 22,35 \\
\hline 8 & Matosinhos & 24 & 1 & 2 & 23,71 & 4,47 & 22,14 \\
\hline 9 & Mateus Leme & 27 & 1 & 2 & 23,49 & 4,31 & 21,98 \\
\hline 10 & Mateus Leme & 36 & 1 & 1 & 23,30 & 4,17 & 21,84 \\
\hline
\end{tabular}

f: valor fenotípico individual.

As alterações de posição quanto à ordenação dos genótipos estão relacionadas a seleção baseada no valor genético, considera o valor genético do caráter em questão, haja vista que, na seleção fenotípica considera-se apenas o dado fenotípico, não considerando o incremento proporcionado pela parcela genética do indivíduo. Observa-se também, um maior ganho com seleção para o método de seleção fenotípica tanto para a variável HT quanto para a variável DAP, devido a principalmente ser considerado o valor fenotípico e não o valor genético do indivíduo.

Salienta-se que, mesmo apresentando maiores ganhos com seleção, a seleção fenotípica pode não proporcionar tais ganhos, pois o fenótipo observado diz respeito a toda variância ambiental e genética (aditiva e dominância), assim, não apresentando uma alta confiabilidade de que aquele fenótipo será expresso em outro local. Mesmo não sendo o melhor método de seleção, a seleção fenotípica ainda apresenta grande importância ao se iniciar um programa de melhoramento genético.

Estes resultados abrem perspectivas para o avanço do programa de melhoramento genético de K. ivorensis, uma vez que é possível observar que dentro da população existe um grande potencial de ganho, que pode ser aumentado ainda mais, realizando cruzamentos entre os melhores indivíduos e/ou inserindo novos indivíduos à esta população, assim aumentando a variabilidade genética e o potencial de ganhos para este caráter.

A heterogeneidade presente em plantios com materiais genéticos de origem seminal, como em testes de procedências, permite o desenvolvimento de genótipos com diferenciados fenótipos, o que possibilita maior ganho na seleção daqueles mais adequados ao objetivo do programa de melhoramento. Azevedo, (2013) e Santos, (2008), citam que testes de procedências constituem em uma estratégia de eficiência comprovada para seleção de genótipos superiores dentro de um programa de melhoramento, pois corroboram com os ganhos genéticos devido a sua considerável heterogeneidade. Dessa forma, tem-se maior facilidade na seleção e os ganhos obtidos são bastante promissores. Garcia e Nogueira, (2005), encontraram resultados relevantes no que tange a estimativas de ganhos genéticos com seleção, chegando a estimativas de ganhos de 36,8\% acima da média da população para a variável volume em Eucalyptus grandis. Isto demonstra o quão promissor podem ser os ganhos ao se selecionar indivíduos superiores dentro de uma população. 
Oliveira et al. - Variabilidade genética de duas procedências de mogno

africano (Khaya ivorensis A. Chev) no cerrado

\section{CONCLUSÕES}

Houveram significativas diferenças, quanto às estimativas dos parâmetros genéticos e no ordenamento dos melhores genótipos entre o modelo que considera os materiais genéticos como fator aleatório e como fixo. A principal diferença entre os modelos analisados está no ganho com seleção, em que a metodologia REML/BLUP considerada o procedimento ótimo, obteve menores ganhos com seleção. Esta metodologia, reduz as predições das médias, assim, podendo atribui-la ao fato destas diferenças possuírem menores efeitos ambientais.

O modelo onde considera-se as progênies como efeito fixo, mostra-se eficiente para seleção inicial de procedências de K. ivorensis. Entretanto, modelos mais robustos onde a seleção é realizada com base nos valores genotípicos individuais e da média da população, devem ser preferidos em populações já submetidas a seleção.

\section{AGRADECIMENTOS}

Os autores agradecem à União Empreendimentos Rurais Ltda e à Universidade Federal de Minas Gerais pelo suporte financeiro.

\section{REFERÊNCIAS BIBLIOGRÁFICAS}

AZEVEDO, L. P. A. Variação genética e seleção para caracteres de crescimento em progênies de Eucalyptus camaldulensis Dehnh. 2013. 61 p. Dissertação (Mestrado) - Universidade Federal de Mato Grosso, Cuiabá, 2013.

BARROS, L. A. G.; SILVA, P. F. R.; PANDOLFI, M. Viabilidade Econômica Da Produção De Mogno-Africano Na Região Sudeste (Khaya ivorensis). SIMTEC, v. 3, n.1, p. 10, 2017.

BERTI, C. L. F. Variação genética, herdabilidades e ganhos na seleção para caracteres de crescimento e forma, em teste de progênies de polinização aberta de Eucalyptus cloeziana, aos 24 anos de idade em Luiz Antônio-SP. 2010. 70 p. Dissertação (Mestre em Agronomia) - Faculdade de Engenharia do Campus de Ilha Solteira, Ilha Solteira, 2010.

CLIMATE-DATA. 2017. Disponível em: <https://pt.climate-data.org/location/24921/>. Acesso em: 31 out. 2017.

COUTO, M. F.; PETERNELLI, L. A.; BARBOSA, M. H. P. Proposta de classificação dos coeficientes de variação para a cultura da cana-de-açúcar. In: REUNIÃO ANUAL DA REGIÃO BRASILEIRA DA SOCIEDADE INTERNACIONAL DE BIOMETRIA, 53., 2008, Lavras. Anais... Lavras: SIB, 2008. CDROM.

FARIAS, P. R. S.; MAIA, P. S. P.; SILVA, A. G.; MONTEIRO, B. S. Ocorrência de Aleurocanthus woglumi em área de reflorestamento com mogno africano na Amazônia Oriental. Revista Ciências Agrárias, Belém, v. 54, n. 1, p. 85-88, 2011.

FIGUEIREDO, E. O; OLIVEIRA, L. C.; BARBOSA, L.K.F. Teca (Tectona grandis L.f.): principais perguntas do futuro empreendedor florestal. Rio Branco: Embrapa Acre, 2005. 87p.

GARCIA, C. H.; NOGUEIRA, M. C. S. N. Utilização da metodologia REML/BLUP na seleção de clones de eucalipto. Scientia Forestalis, Piracicaba, n.68, p.107-112, 2005.

GOMES, D. M. Análise de viabilidade técnica, econômico-financeiro para implantação da cultura do mogno africano (Khaya ivorensis A. Chev.) na região oeste de Minas Gerais. 2010. 70 p. Trabalho de Conclusão de Curso (Especialização em Gestão Florestal) - Universidade Federal do Paraná, Curitiba, 2010.

IBÁ - INDUSTRIA BRASILEIRA DE ÁRVORES (IBÁ). Relatório IBÁ 2017: ano base 2016. Brasília: IBA, 2017. Disponível em < http://iba.org/images/shared/Biblioteca/IBA_RelatorioAnual2017.pdf>. Acesso em: 09 nov. 2017.

IBF - INSTITUTO BRASILEIRO DE FLORESTAS. O Apagão Florestal - Produção de madeira da Amazônia pode cair 64\% e demanda deve quadruplicar até 2030. 2017a. Disponível em: < https://www.ibflorestas. org.br/noticias/850-o-apagao-florestal-producao-de-madeira-da-amazonia pode-cair-64-e-demanda-devequadruplicar-ate-2030.html >. Acesso em: 02 nov. 2017. 
IBF - INSTITUTO BRASILEIRO DE FLORESTAS. Mogno Africano - plantio comercial no Brasil. $2017 \mathrm{~b}$. Disponível em: < https://www.ibflorestas.org.br/noticias/922-mogno-africano-plantio-comercial-no-brasil. html>. Acesso em: 02 nov. 2017.

LONDRES, F. Agrotóxicos no Brasil: um guia para ação em defesa da vida. Rio de Janeiro: PTA - Assessoria e Serviços a Projetos em Agricultura Alternativa, 2011. 190 p.

MARTINEZ, D. T.; RESENDE, M. D. V.; COSTA, R. B.; HIGA, A. R.; SANTOS, G. A.; FIER, I. S. N. Estudo da interação genótipo $\mathrm{x}$ ambiente em progênies de Pinus taeda por meio da análise de parâmetros genéticos. Floresta, Curitiba, v. 42, n. 3, p. 539 - 552, 2012.

NATIVIDADE, G. S. Análise do cenário da produção de mogno africano (Khaya ivorensis) no cerrado. 2016. 45 p. Trabalho de conclusão de curso (Bacharelado em Gestão de Agronegócios) - Universidade de Brasília, Brasília, 2016.

NOVICK, R. R.; LEMES, M R.; NAVARRO, C.; CACCONE, A.; BERMINGHAM, E. Genetic structure of Mesoamerican populations of Big-leaf mahogany (Swietenia macrophylla) inferred from microsatellite analysis. Molecular Ecology, v. 12, n. 11, p. 2885-93, 2003.

RESENDE, M. D. V. Selegen-Reml/Blup: Sistema Estatístico e Seleção Genética Computadorizada via Modelos Lineares Mistos. Colombo: Embrapa Florestas, 2007. 360 p.

RESENDE, M. D. V.; DUARTE, J. B. Precisão e controle de qualidade em experimentos de avaliação de cultivares. Pesquisa Agropecuária Tropical, Goiânia, v. 37, n. 3, p. 182-194, 2007.

RESENDE, M. D. V. Genética biométrica e estatística no melhoramento de plantas perenes. Brasília: Embrapa Informação Tecnológica, 2002. 975 p.

ROSA, F. O. Zoneamento edafoclimático e respostas do mogno africano às condições do cerrado. 2014. 85 p. Dissertação (Mestrado em Agronomia) - Universidade Federal de Goiás, Goiânia, 2014.

ROSADO, A. M.; ROSADO, T. B.; RESENDE, M. F. R.; BHERING, L. L.; CRUZ, C. D. Ganhos genéticos preditos por diferentes métodos de seleção em progênies de Eucalyptus uroplhylla. Pesquisa Agropecuária Brasileira. Brasília, v. 44, n. 12, p. 1653-1659, 2009.

SAMPAIO, P. T. B.; RESENDE, M. D. V.; ARAÚJO, A. J. Estimativas de parâmetros genéticos e métodos de seleção para o melhoramento de Pinus caribaea var. hondurensis. Pesquisa Agropecuária Brasileira. Brasília, v. 35, n. 11, 11.p, 2000.

SANTOS, A. M. Estimativas de parâmetros genéticos e avaliação da eficiência de seleção precoce em baru (Dipteryx alata Vog.). 2008. 91 p. Dissertação (Mestrado em Ciências Florestais) - Universidade Federal de Lavras, Lavras 2008.

SILVA, L. E. Variabilidade e estimação de parâmetros genéticos via modelos mistos em canafístula. 2015. 54 p. Dissertação (Mestrado em Agronomia) - Universidade Federal da Grande Dourados, Dourados, 2015.

SILVA, L. V. M. S. Propriedades físicas e mecânicas da madeira de mogno africano (Khaya ivorensis A. Chev). 2013. 27 p. Trabalho de conclusão de curso (Graduação em Engenharia Florestal) - Instituto Florestas, Universidade Federal Rural do Rio de Janeiro, Seropédica, 2013.

SOARES, S. D. Diversidade genética em população de melhoramento de mogno africano (Khaya ivorensis A. Chev.). 2014. 69 p. Dissertação (Mestrado em Genética e Biologia Molecular) - Universidade Federal de Goiás, Goiânia, 2014.

TEIXEIRA, V. C. M. Avaliação da usinagem da madeira de mogno africano (Khaya ivorensis A. Chev.). 2011. 45 p. Trabalho de conclusão de curso (Graduação em Engenharia Florestal) - Instituto de Florestas, Universidade Federal Rural do Rio de Janeiro, Seropédica, 2011.

Recebido em: 30/05/2018

Disponível em: 14/02/2019

Sci. For., Piracicaba, v. 47, n. 124, p. 624-631, dez. 2019

DOI: doi.org/10.18671/scifor.v47n124.04 